Journal of Applied Mathematics and Stochastic Analysis, 16:3 (2003), 249-255.

Printed in the USA (C)2003 by North Atlantic Science Publishing Company

\title{
REAL ZEROS OF CLASSES OF RANDOM ALGEBRAIC POLYNOMIALS
}

\author{
K. FARAHMAND \\ University of Ulster \\ Department of Mathematics \\ Jordanstown Co. Antrim BT37 OQB, UK \\ M. SAMBANDHAM \\ Morehouse College \\ Department of Mathematics \\ Atlanta, GA 30114 USA
}

(Received October, 2002; Revised March, 2003)

\begin{abstract}
There are many known asymptotic estimates for the expected number of real zeros of an algebraic polynomial $a_{0}+a_{1} x+a_{2} x^{2}+\cdots+a_{n-1} x^{n-1}$ with identically distributed random coefficients. Under different assumptions for the distribution of the coefficients $\left\{a_{j}\right\}_{j=0}^{n-1}$ it is shown that the above expected number is asymptotic to $O(\log n)$. This order for the expected number of zeros remains valid for the case when the coefficients are grouped into two, each group with a different variance. However, it was recently shown that if the coefficients are non-identically distributed such that the variance of the $j$ th term is $\left(\begin{array}{l}n \\ j\end{array}\right)$ the expected number of zeros of the polynomial increases to $O(\sqrt{n})$. The present paper provides the value for this asymptotic formula for the polynomials with the latter variances when they are grouped into three with different patterns for their variances.
\end{abstract}

Keywords. Number of Real Zeros, Random Algebraic Polynomials, Kac-Rice Formula, Random Variables, Binomial Coefficients.

AMS (MOS) subject classification: 60G99, 60H99.

\section{Introduction}

A random algebraic polynomial defined as

$$
P_{n}(x)=\sum_{j=0}^{n-1} a_{j} x^{j}
$$

where the coefficients $\left\{a_{j}\right\}_{j=0}^{n-1}$ are a sequence of normally distributed random variables is well studied. In particular the mathematical behaviour of the number of real zeros, 
$N_{n}(a, b)$ in the interval $(a, b)$ is interesting and somehow unexpected. For the case of identically distributed coefficients with mean zero it is known that the mathematical expected number of real zeros, denoted by $E N_{n}(-\infty, \infty)$ is asymptotic to $(2 / \pi) \log n$, see Kac [5] or the recent work of Wilkins [10]. This asymptotic value remains invariant for all other cases of identically distributed coefficients belonging to the domain of attraction of normal law. For other distributions outside this domain Logan and Shepp [6] and [7] have shown that there is a slight increase in the number of real zeros, when they considered the cases which include Cauchy distributed coefficients.

However, Edelman and Kostlan [2] in their expository work have shown that the expectation $E N_{n}(-\infty, \infty)$ increases significantly, to $\sqrt{n}$, when they assume a special non- identical distribution for the coefficients. They chose normal distribution for the coefficients $a_{j}$ with mean zero and variance $\left(\begin{array}{l}n \\ j\end{array}\right)$. This particular choice of variances is of special interest for their physical properties investigated by Ramponi [8] and indeed their mathematical behaviour. For the latter, this choice permits the variances of the coefficients to be small for the first few and the last few terms of the polynomials compared with the middle terms. It is presumably the case that, under some mild assumptions, the Edelman and Kostlan results could be generalized to remain valid for other forms of variances for $a_{j}$ rather than just $\left(\begin{array}{l}n \\ j\end{array}\right)$. These authors, however, were unable to make any substantial progress toward this conjecture.

Instead in this work for $\sigma_{1}^{2}, \sigma_{2}^{2}$ and $\sigma_{3}^{2}$ all being absolute constants and $n_{1}$ and $n-n_{1}$ being a proportion of $n$ we assume

$$
\operatorname{var}\left(a_{j}\right)= \begin{cases}\sigma_{1}^{2}\left(\begin{array}{c}
n_{1}-1 \\
j
\end{array}\right) & 0 \leq j \leq n_{1}-1 \\
\sigma_{2}^{2} & n_{1} \leq j \leq n-n_{1}-1 \\
\sigma_{3}^{2}\left(\begin{array}{ll}
n-1-1 \\
n-j-1
\end{array}\right) & n-n_{1} \leq j \leq n-1 .\end{cases}
$$

Our theorem shows that for these classes of distribution we are still able to obtain a significant number of zeros. As with the above assumptions the variance of the coefficients in $P_{n}(x)$ is not necessarily larger for the middle terms obeying the above pattern for the conjecture, and still being able to obtain a significantly large number of real zeros, the assumption for the above conjecture should be outside (1.2).

Also in [4] the corresponding result for varying the means of the coefficients, rather than variances, is studied. The earlier works on random polynomials are reviewed in the comprehensive book by Bharucha-Reid and Sambandham [1] and more recently in Farahmand [3]. We prove:

Theorem 1.1: Let the coefficients $\left\{a_{j}\right\}_{j=0}^{n-1}$ of $P_{n}(x)$ be normally distributed with mean zero and variances given in (1.2), when $n_{1}$ (and therefore $n-n_{1}$ ) is a proportion of $n$, that is for $k>1$ an absolute constant we have $n_{1}=n / k$. Then the expected number of real zeros of $P_{n}(x)$ is

$$
E N_{n}(-\infty, 0) \sim E N_{n}(0, \infty) \sim \frac{\sqrt{n_{1}-1}}{2}
$$

The proof of our theorem also reveals another interesting behaviour for the expected number of real zeros. In the interval $(-1,1)$ this behaviour is dictated by the variance of the first few $n_{1}$ terms. However, outside this interval the behaviour is dictated by the variance of the last few terms. The variance of the middle terms is not supported by the binomial terms, and therefore could not influence $E N_{n}(-\infty, \infty)$. 


\section{Primary Analysis for the moments.}

In order to estimate the expected number of real zeros of the random polynomial we need to evaluate first the variances of $P_{n}(x)$ and its derivative $P_{n}^{\prime}(x)$. To this end we assume $x \in(0,1)$, then since the coefficients of $P_{n}(x)$ are independent with mean zero and their variances are given in (1.2) we can say

$$
\begin{aligned}
A^{2}= & \operatorname{var}\left(P_{n}(x)\right)=\sigma_{1}^{2} \sum_{j=0}^{n_{1}-1} x^{2 j}\left(\begin{array}{c}
n_{1}-1 \\
j
\end{array}\right)+\sigma_{2}^{2} \sum_{j=n_{1}}^{n-n_{1}-1} x^{2 j} \\
& +\sigma_{3}^{2} \sum_{j=n-n_{1}}^{n-1} x^{2 j}\left(\begin{array}{c}
n_{1}-1 \\
j-n+n_{1}
\end{array}\right) .
\end{aligned}
$$

Using the well known identity for the first and second term on the right hand side of (2.1) and since $\left(\begin{array}{c}n_{1}-1 \\ j-n_{1}+n_{1}\end{array}\right)=\left(\begin{array}{c}n_{1}-1 \\ n-j-1\end{array}\right)$ from (2.1) we can obtain

$$
\begin{aligned}
A^{2}= & \sigma_{1}^{2}\left(x^{2}+1\right)^{n_{1}-1}+\sigma_{2}^{2}\left\{\frac{x^{2\left(n_{1}-1\right)}-x^{2\left(n-n_{1}\right)}}{1-x^{2}}\right\} \\
& +\sigma_{3}^{2} \sum_{j=n-n_{1}}^{n-1} x^{2 j}\left(\begin{array}{c}
n_{1}-1 \\
j-n+n_{1}
\end{array}\right) .
\end{aligned}
$$

The last term of (2.2) can be evaluated further as

$$
\sigma_{3}^{2} \sum_{j=0}^{n_{1}-1} x^{2\left(j+n-n_{1}\right)}\left(\begin{array}{c}
n_{1}-1 \\
j
\end{array}\right)=\sigma_{3}^{2} x^{2\left(n-n_{1}\right)}\left(x^{2}+1\right)^{n_{1}-1} .
$$

Now we first assume $0<x<1-\epsilon$ where $\epsilon \equiv \epsilon_{n}=n^{-\lambda}$ and $\lambda$ has a positive value dependent on $n$ only given by

$$
\lambda=1-\frac{\log \log n^{k}}{\log n},
$$

where $k$ is any constant number greater than two. Now let $\eta=\min \left\{n_{1},\left(n-n_{1}\right)\right\}$. By our assumption of $n_{1}$, obviously $\eta=n / c$ for some constant $c$. Therefore we can show that the terms in the forms $x^{\left(2 n_{1}-1\right)}, x^{2\left(n-n_{1}\right)}$ and $x^{2 n}$ which appeared in $(2.2)$ and (2.3) are small and can be ignored in the estimation. To this end, since for this range of $x$ all of these terms are smaller than $x^{2 \eta}$, for all sufficiently large $n$ we can write

$$
\begin{aligned}
\max \left\{x^{n}, x^{n_{1}}, x^{\left(n-n_{1}\right)}\right\} & \leq x^{\eta} \\
& <(1-\epsilon)^{\eta}=\left(1-n^{-\lambda}\right)^{\eta} \\
& =\exp \left(-\eta n^{-\lambda}\right)=\exp \left(\frac{-n^{1-\lambda}}{c}\right) \\
& \sim n^{-k} .
\end{aligned}
$$

The last equation in (2.5) is obtained using the definition of $\lambda$ in (2.4) and the fact that $c$ is a positive constant. In what follows, in order to simplify the analysis, we assume 
$k=10$. However any choice of $k>2$ leads to the result. From (2.2) and (2.3) and since $\sigma_{2}^{2}$ and $\sigma_{3}^{2}$ are constants we can see

$$
A^{2}=\sigma_{1}^{2}\left(x^{2}+1\right)^{n_{1}-1}\left\{1+o\left(n^{-10}\right)\right\} .
$$

We now proceed to obtain the covariance of $P(x)$ and its derivative $P^{\prime}(x)$. Since the coefficients of the polynomial are independent with mean zero we can obtain

$$
\begin{aligned}
C= & \operatorname{cov}\left\{P(x), P^{\prime}(x)\right\}=E\left(\sum_{j=0}^{n-1} a_{j} x^{j} \sum_{j=1}^{n-1} j a_{j} x^{j-1}\right) \\
= & \sum_{j=1}^{n-1} j x^{2 j-1} \operatorname{var}\left(a_{j}\right) \\
= & \sigma_{1}^{2} \sum_{j=1}^{n_{1}-1} j x^{2 j-1}\left(\begin{array}{c}
n_{1}-1 \\
j
\end{array}\right)+\sigma_{2}^{2} \sum_{j=n_{1}}^{n-n_{1}-1} j x^{2 j-1} \\
& +\sigma_{3}^{2} \sum_{j=n-n_{1}}^{n-1} j x^{2 j-1}\left(\begin{array}{c}
n_{1}-1 \\
n-j-1
\end{array}\right) .
\end{aligned}
$$

Since $\left(\begin{array}{c}n_{1}-1 \\ n-j-1\end{array}\right)=\left(\begin{array}{c}n_{1}-1 \\ j-n+n_{1}\end{array}\right)$ the last term which appears in (2.7) is equal to

$$
\sigma_{3}^{2} \sum_{j=0}^{n_{1}-1}\left(j+n-n_{1}\right) x^{2\left(j+n-n_{1}\right)-1}\left(\begin{array}{c}
n_{1}-1 \\
j
\end{array}\right) .
$$

The closed form of the above sum can be obtained by simple differentiation of the well known formula for the binomial sum. This together with (2.7) yields

$$
\begin{aligned}
C= & \sigma_{1}^{2}\left(n_{1}-1\right) x\left(x^{2}+1\right)^{n_{1}-2}+\sigma_{2}^{2}\left\{\frac{x^{2 n_{1}+1}-x^{2\left(n-n_{1}\right)+1}}{\left(1+x^{2}\right)^{2}}\right. \\
& \left.+\frac{n_{1} x^{2 n_{1}-1}-\left(n-n_{1}\right) x^{2\left(n-n_{1}\right)-1}}{1-x^{2}}\right\} \\
& +\sigma_{3}^{2}\left(n-n_{1}\right)\left(n_{1}-1\right) x^{2\left(n-n_{1}\right)+1}\left(x^{2}+1\right)^{n_{1}-2} .
\end{aligned}
$$

Now since for $0<x<1-\epsilon$ we can use the inequality obtained in (2.6) for (2.8) to obtain

$$
\begin{aligned}
C & =\left(n_{1}-1\right) x\left(x^{2}+1\right)^{n_{1}-2}\left\{\sigma_{1}^{2}+\sigma_{3}^{2}\left(n-n_{1}\right) x^{2\left(n-n_{1}\right)}\right\}+o\left(n^{-8}\right) \\
& =\sigma_{1}^{2}\left(n_{1}-1\right) x\left(x^{2}+1\right)^{n_{1}-2}\left\{1+o\left(n^{-8}\right)\right\} .
\end{aligned}
$$

Finally we derive the variance of $P^{\prime}(x)$. Using the above assumption for the coefficients of the polynomial we have

$$
\begin{aligned}
B^{2}= & \operatorname{var}\left(P^{\prime}(x)\right)=\sigma_{1}^{2} \sum_{j=0}^{n_{1}-1} j^{2} x^{2 j-2}\left(\begin{array}{c}
n_{1}-1 \\
j
\end{array}\right) \\
& +\sigma_{2}^{2}\left\{\sum_{j=0}^{n-n_{1}-1} j^{2} x^{2 j-2}-\sum_{j=0}^{n_{1}-1} j^{2} x^{2 j-2}\right\}
\end{aligned}
$$




$$
+\sigma_{3}^{2} \sum_{j=n-n_{1}}^{n-1} j^{2} x^{2 j-2}\left(\begin{array}{c}
n_{1}-1 \\
j-n-n_{1}
\end{array}\right) .
$$

Now for this range of $x$

$$
\begin{aligned}
\sum_{j=0}^{n_{1}-1} j^{2} x^{2 j-2}= & \left\{x^{2}+1-x^{2 n_{1}+2}-x^{2 n_{1}}-n^{2} x^{2 n_{1}+2}+2 n^{2} x^{2 n_{1}}\right. \\
& \left.-n^{2} x^{2 n_{1}-2}+2 n x^{2 n_{1}+2}-2 n_{1} x^{2 n_{1}}\right\}\left(1-x^{2}\right)^{-3} \\
= & \frac{1+x^{2}}{\left(1-x^{2}\right)^{3}}\left\{1+o\left(n_{1}^{-18}\right)\right\} .
\end{aligned}
$$

Also for all $x$

$$
\sum_{j=0}^{n_{1}-1} j^{2} x^{2 j-2}\left(\begin{array}{c}
n_{1}-1 \\
j
\end{array}\right)=\left(n_{1}-1\right)\left(x^{2}+1\right)^{n_{1}-3}\left(n_{1} x^{2}-x^{2}+1\right)
$$

and

$$
\begin{aligned}
\sum_{j=n-n_{1}}^{n-1} j^{2} x^{2 j-2}\left(\begin{array}{c}
n_{1}-1 \\
j-n-n_{1}
\end{array}\right)= & x^{2\left(n-n_{1}\right)}\left\{\left(n-n_{1}\right)^{2} \sum_{j=0}^{n_{1}-1} x^{2 j-2}\left(\begin{array}{c}
n_{1}-1 \\
j
\end{array}\right)\right. \\
& +2\left(n-n_{1}\right) \sum_{j=0}^{n_{1}-1} j x^{2 j-2}\left(\begin{array}{c}
n_{1}-1 \\
j
\end{array}\right) \\
& \left.+\sum_{j=0}^{n_{1}-1} j^{2} x^{2 j-2}\left(\begin{array}{c}
n_{1}-1 \\
j
\end{array}\right)\right\}
\end{aligned}
$$

Therefore from (2.10)-(2.13) and a little algebra we obtain

$$
B^{2}=\sigma_{1}^{2}\left(n_{1}-1\right)\left(x^{2}+1\right)^{n_{1}-3}\left(n_{1} x^{2}-x^{2}+1\right)\left\{1+o\left(n^{-18}\right)\right\} .
$$

\section{Proof of theorem.}

Now we use the well known Kac-Rice formula for the expected number of real roots obtained in Kac [5] and Rice [9]. From [3, page 32] the expected number of real roots of $P_{n}(x)$ in $(a, b)$ is given by

$$
E N_{n}(a, b)=\left(\frac{1}{\pi}\right) \int_{a}^{b}\left(\frac{\Delta}{A^{2}}\right) d x
$$

where $\Delta^{2}=A^{2} B^{2}-C^{2}$. Since from (2.6), (2.9) and (2.14), for all sufficiently large $n_{1}$,

$$
\Delta^{2} \sim \sigma_{1}^{2}\left(n_{1}-1\right)\left(x^{2}+1\right)^{2 n_{1}-4}
$$

so from (2.6) we can write

$$
\begin{aligned}
E N_{n}(0,1-\epsilon) & =\int_{0}^{1-\epsilon} \frac{\Delta}{\pi A^{2}} d x \\
& \sim \frac{\sqrt{n_{1}-1}}{\pi} \int_{0}^{1-\epsilon} \frac{d x}{x^{2}+1} \\
& \sim \frac{(\pi-\epsilon) \sqrt{n_{1}-1}}{4 \pi} \sim \frac{\sqrt{n_{1}-1}}{4}
\end{aligned}
$$


In the following we show that in the interval $(1-\epsilon, 1)$ the expected number of real zeros is small compared with (3.2) and therefore can be ignored. To this end from the definitions of $A^{2}, B^{2}$ and $C$ given in (2.1), (2.7) and (2.10) we note that

$$
\begin{aligned}
B^{2} & =\operatorname{var}\left(P^{\prime}(x)\right)=\operatorname{var}\left(\sum_{j=0}^{n-1} a_{j} j x^{j-1}\right) \\
& =\sum_{j=0}^{n-1} j^{2} \operatorname{var}\left(a_{j} x^{j-1}\right) \\
& <n^{2} \sum_{j=0}^{n-1} \operatorname{var}\left(a_{j} x^{j-1}\right),
\end{aligned}
$$

and therefore

$$
\begin{aligned}
\frac{\Delta}{A^{2}} & <\frac{B}{A}<\sqrt{\frac{n^{2} \sum_{j=0}^{n-1} \operatorname{var}\left(a_{j} x^{j-1}\right)}{\sum_{j=0}^{n-1} \operatorname{var}\left(a_{j} x^{j}\right)}} \\
& =\sqrt{\frac{n^{2}}{x^{2}}}<\frac{n}{1-\epsilon} .
\end{aligned}
$$

Hence from (3.3) and the Kac-Rice formula and since from the definition of $\epsilon$ we can see $n \epsilon=n^{\left(\log \log n^{10} / \log n\right)}=\log n^{10}$ and $\epsilon=n^{-\lambda}=\log n^{10} / n$ which tends to zero as $n \rightarrow \infty$

$$
E N_{n}(1-\epsilon, 1) \leq \int_{1-\epsilon}^{1} \frac{B}{A} d x<\frac{n \epsilon}{1-\epsilon}=o\left(\log n^{10}\right) .
$$

This together with (3.2) yields

$$
E N_{n}(0,1) \sim \frac{\sqrt{n_{1}-1}}{4} .
$$

Now we use this result obtained for the interval $(0,1)$ to derive the result for the entire interval $(-\infty, \infty)$. To this end we first note that changing $x$ to $-x$ leaves the distribution of the coefficients invariant. Therefore $E N(-\infty, 0) \sim E N(0, \infty)$. Also in order to obtain the result for the interval $(1, \infty)$ we replace $x$ by $1 / x$ to obtain

$$
P_{n}\left(\frac{1}{x}\right)=\sum_{j=0}^{n-1} a_{j} x^{-j}=x^{-n+1} \sum_{j=0}^{n-1} a_{j} x^{n-j-1} .
$$

The above polynomial has the same properties as $P_{n}(x)$ if we exchange the order of coefficients in the latter. Therefore the above proof presented for $(0,1)$ remains valid by only changing the order of coefficients.

\section{References}

[1] Bharucha-Reid, A.T. and Sambandham, M., Random Polynomials, Academic Press, New York 1986.

[2] Edelman, A. and Kostlan, E., How many zeros of a random polynomial are real?, Bull. Amer. Math. Soc. 32 (1995), 1-37. 
[3] Farahmand, K., Topics in Random Polynomials, Addison Wesley Longman, London 1998.

[4] Farahmand, K., Flood, P. and Hannigan, P., Zeros of a random algebraic polynomial with coefficient means in geometric progression, J. Math. Anal. Appl. 269 (2002), 137-148.

[5] Kac, M., On the average number of real roots of a random algebraic equation, Bull.Amer.Math.Soc. 49 (1943), 314-320.

[6] Logan, B.F. and Shepp, L.A., Real zeros of random polynomials, Proc. London Math. Soc. 18 (1968), 29-35.

[7] Logan, B.F. and Shepp, L.A., Real zeros of random polynomials. II, Proc. London Math. Soc. 18 (1968),308-314.

[8] Ramponi, A., A note on the complex roots of complex random polynomials, Statistics and Prob. Lett., 44 (1999), 181-187.

[9] Rice, S.O., Mathematical theory of random noise, Bell. System Tech. J. 25 (1945), 46156. Reprinted in: Selected Papers on Noise And Stochastic Processes (ed. N. Wax), Dover, New York, 1954, 133-294.

[10] Wilkins, J.E., An asymptotic expansion for the expected number of real zeros of a random polynomial, Proc. Amer. Math. Soc. 103 (1988), 1249- 1258. 


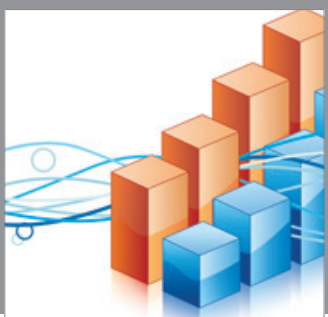

Advances in

Operations Research

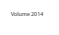

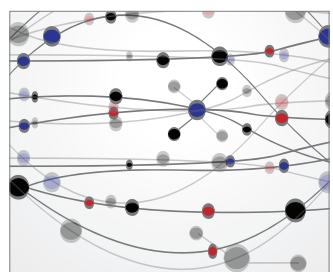

\section{The Scientific} World Journal
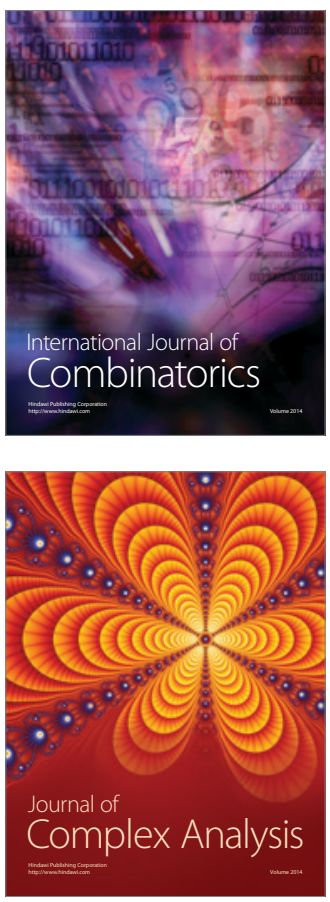

International Journal of

Mathematics and

Mathematical

Sciences
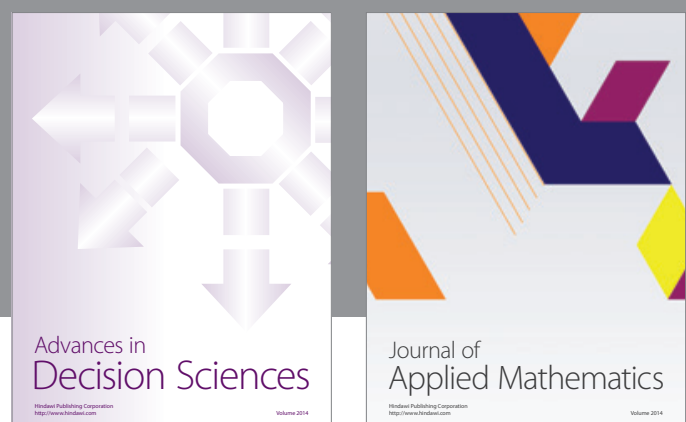

Journal of

Applied Mathematics
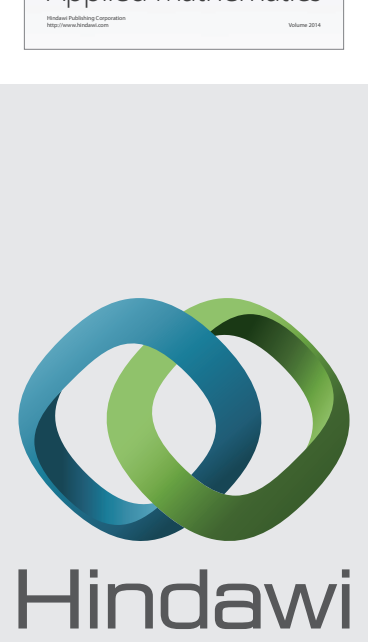

Submit your manuscripts at http://www.hindawi.com
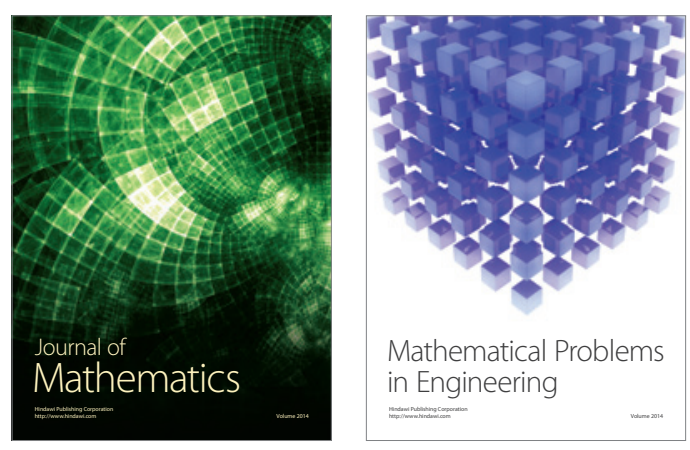

Mathematical Problems in Engineering
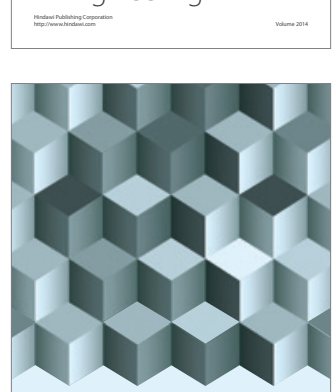

Journal of

Function Spaces
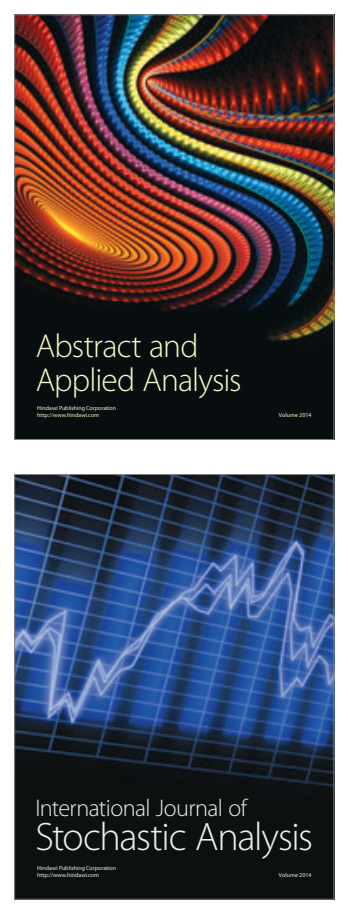

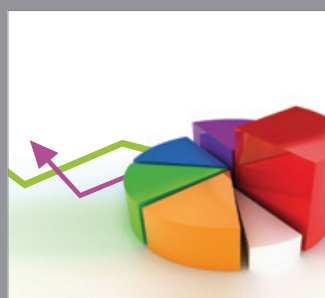

ournal of

Probability and Statistics

Promensencen
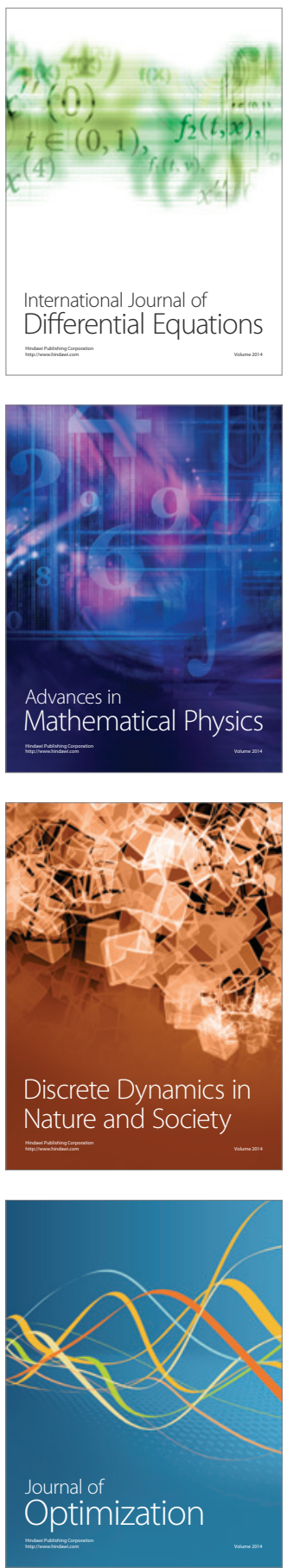